\title{
Duality in Noncommutative Maxwell-Chern-Simons Theory
}

\author{
Victor O. Rivelles* \\ Instituto de Física, Universidade de São Paulo \\ Caixa Postal 66318, 05315-970, São Paulo, SP, Brazil \\ E-mail: rivelles@fma.if.usp.br
}

Applying a master action technique we obtain the dual of the noncommutative Maxwell-ChernSimons theory. The equivalence between the Maxwell-Chern-Simons theory and the self-dual model in commutative space-time does not survive in the non-commutative setting. We also point out an ambiguity in the Seiberg-Witten map.

Fifth International Conference on Mathematical Methods in Physics - IC2006 April 24-28 2006

Centro Brasileiro de Pesquisas Fisicas, Rio de Janeiro, Brazil

*Speaker. 
I will report on a work done in collaboration with E. Harikumar about duality in noncommutative theories in three dimensions [1]. The generalization of the well known equivalence between the Maxwell-Chern-Simons (MCS) theory and the self-dual (SD) model [2] to NC space-time was investigated in [1]. The master action technique, which was used to establish the equivalence between these models in commutative space-time, has been adopted in [3] and [4] and these authors have reached different conclusions regarding the equivalence in the NC setting. In [4], after eliminating some of the fields from the master action, the perturbative solution to the field equations were used and it was argued that the NCMCS theory is equivalent to the NCSD model when the Chern-Simons (CS) term has a cubic contribution like in the non-Abelian case. In [3], however, which also used the master action method, it was argued that the NCMCS theory constructed by applying the inverse Seiberg-Witten (SW) map [5], is equivalent to a theory where the cubic interaction of the vector field is absent in the CS term. A different approach to study the equivalence has been adopted in [6]. Using an iterative embedding method [7] for the NCSD model, a dual equivalent theory was constructed to all orders in the NC parameter. This dual model differs from NCMCS theory in the coefficient of the cubic interaction of the CS term and this breaks gauge invariance. In [8], the SW mapped NCMCS theory was argued to be equivalent to a theory where the effect of noncommutativity appears through a non-covariant term. This term vanishes in the commutative limit and the SD model is then recovered. It is then imperative, using alternative approaches, to reexamine the relation between NCMCS theory and NCSD model since the previous studies are inconclusive. Also, this result has interesting implications for deriving the bosonization rules for the NC massive Thirring model [3, 4].

Here we will use a procedure which was applied to get a dual description of the sigma model [9] and was also used recently to show the equivalence between massive Abelian gauge theories in $3+1$ dimensions [10]. We first apply the procedure to the partition function of the SW mapped NCMCS theory to order $\theta$ and derive the dual theory also to order $\theta$. We then argue that this result can be extended to all orders in $\theta$. From the dual theory constructed, we show that the equivalence between the MCS theory and the SD model do not get generalized to the NC setting. In our way to derive the SW map for the NCMCS theory we found that the presence of a massive coupling constant turns the map ambiguous. An infinite number of terms can be present in the map but we choose the minimal set required by the map.

\section{Ambiguity in the Seiberg-Witten Map}

The SW map is obtained by requiring that an ordinary gauge transformation on $A_{\mu}$ with parameter $\lambda$ is equivalent to a NC gauge transformation on $\hat{A}_{\mu}$ with gauge parameter $\hat{\lambda}$ so that ordinary gauge fields that are gauge equivalent are mapped into NC gauge fields that are also equivalent. In four dimension, where it was originally derived, the SW map for the Abelian gauge theory to first order in $\theta$ is given by

$$
\begin{aligned}
\hat{A}_{\mu} & =A_{\mu}-\frac{1}{2} \theta^{\alpha \beta} A_{\alpha}\left(2 \partial_{\beta} A_{\mu}-\partial_{\mu} A_{\beta}\right), \\
\hat{\lambda} & =\lambda+\frac{1}{2} \theta^{\alpha \beta} \partial_{\alpha} \lambda A_{\beta} .
\end{aligned}
$$


The NC action, when expanded to first order in $\theta$,

$$
\hat{S}=-\frac{1}{4} \int d^{4} x \hat{f}^{\mu v}\left(\hat{f}_{\mu v}+2 \theta^{\alpha \beta} \partial_{\alpha} \hat{A}_{\mu} \partial_{\beta} \hat{A}_{v}\right)
$$

with $\hat{f}_{\mu \nu}=\partial_{\mu} \hat{A}_{\nu}-\partial_{v} \hat{A}_{\mu}$, gives rise to the SW action

$$
S_{S W}=-\frac{1}{4} \int d^{4} x\left[f^{2}+2 \theta^{\alpha \beta}\left(f^{\mu v} f_{\mu \alpha} f_{v \beta}-\frac{1}{4} f_{\alpha \beta} f^{2}\right)\right] .
$$

The question we are interested in is the freedom allowed by the SW map. Due to its nature we can add to the map (1.1) any gauge invariant term built with $\theta$ and derivatives of the gauge field with the right dimension and the new map will still be a SW map. The question is then how the SW action will be affected. To answer this question let us note that by adding to the map (1.1) a term like

$$
\delta \hat{A}_{\mu}=\theta^{\alpha \beta} T_{\mu \alpha \beta},
$$

we get a contribution to the action (1.4) like

$$
\delta \hat{S}=-\int d^{4} x \theta^{\alpha \beta} f^{\mu v} \partial_{\mu} T_{v \alpha \beta}
$$

Then if this integral vanishes we will not get any new contribution to the SW action. Since in four dimensions the gauge field has dimension one the only gauge invariant terms we can add to the SW map have $T_{\mu \alpha \beta}$ of the form $\partial_{\mu} f_{\alpha \beta}, \partial_{\alpha} f_{\mu \beta}$ and $\partial^{\rho} f_{\rho \beta} \eta_{\alpha \mu}$. The first term is a gauge transformation to order $\theta$ [11] and gives no contribution to the SW action. The second one is proportional the first after applying the Bianchi identity. Finally, the third term gives no contribution to the action since the integral in (1.6) vanishes. Then the SW map to order $\theta$ is essentially unique in four dimensions. However, as we shall see, in three dimensions the situation is completely different.

In three dimensions the NCMCS theory is described by the Lagrangian

$$
\hat{\mathscr{L}}_{N C M C S}=-\frac{1}{4 g^{2}} \hat{F}_{\mu v} * \hat{F}^{\mu v}+\frac{\mu}{2} \varepsilon_{\mu v \lambda} \hat{A}^{\mu} *\left(\hat{F}^{v \lambda}+\frac{2 i}{3} \hat{A}^{v} * \hat{A}^{\lambda}\right)
$$

where $\hat{F}_{\mu \nu}=\partial \hat{A}_{\mu}-\partial \hat{A}_{v}-i\left[\hat{A}_{\mu}, \hat{A}_{v}\right]_{*}$ while the NCSD model with a compensating Stückelberg field has a Lagrangian given by

$$
\hat{\mathscr{L}}_{N C S D}=\frac{g^{2}}{2}\left(\hat{f}_{\mu}-\hat{b}_{\mu}\right) *\left(\hat{f}^{\mu}-\hat{b}^{\mu}\right)-\frac{1}{2 k} \varepsilon_{\mu v \lambda} \hat{f}^{\mu} *\left(\partial^{v} \hat{f}^{\lambda}-\frac{2 i}{3} \hat{f}^{v} * \hat{f}^{\lambda}\right)
$$

where $\hat{b}_{\mu}=i \hat{\mathscr{U}}^{-1} * \partial_{\mu} \hat{\mathscr{U}}, \hat{\mathscr{U}} \in U(1)$. The NCMCS theory is invariant under the $U(1)$ gauge transformation

$$
\hat{A}_{\mu} \rightarrow \hat{U}^{-1} * \hat{A}_{\mu} * \hat{U}+i \hat{U}^{-1} * \partial_{\mu} \hat{U}
$$

while the NC Stückelberg-SD Lagrangian is invariant under

$$
\begin{aligned}
\hat{f}_{\mu} & \rightarrow \hat{U}^{-1} * \hat{f}_{\mu} * \hat{U}+i \hat{U}^{-1} * \partial_{\mu} \hat{U} \\
\hat{\mathscr{U}} & \rightarrow \hat{\mathscr{U}} * \hat{U} .
\end{aligned}
$$

We should remark that for the pure NCCS theory the SW map has the form (1.1) if the CS coefficient $\mu$ is chosen to be dimensionless so that the gauge field has dimension one. The pure 
NCCS theory has the remarkable property that the SW action has no dependence whatsoever in $\theta$ [12].

In the NCMCS theory and NCSD model the situation is rather different since one of the couplings must be dimensionfull and this choice determines the gauge field dimensionality. If we make the usual choice for the gauge field dimensionality to be one then $g^{2}$ in the NCMCS theory has dimension one and we can use the SW map (1.1) to obtain

$$
\mathscr{L}_{S W}=-\frac{1}{4 g^{2}}\left[F_{\mu v} F^{\mu v}+2 \theta^{\alpha \beta} F_{\alpha \mu} F_{\beta v} F^{\mu v}-\frac{1}{2} \theta^{\alpha \beta} F_{\alpha \beta} F_{\mu v} F^{\mu v}\right]+\frac{\mu}{4} \varepsilon^{\mu \nu \lambda} A_{\mu} F_{v \lambda} .
$$

The fact that $g^{2}$ has dimension one means now that the SW map (1.1) has an arbitrariness since we can add an infinite number of gauge invariant terms, all linear in $\theta$, but with different powers of derivatives of $F_{\mu \nu}$. These arbitrary terms in the SW map have the form $g^{6} \theta^{\alpha \beta} T_{\mu \alpha \beta}$ where the $g^{6}$ factor was chosen so that $T_{\mu \alpha \beta}$ is a dimensionless function of $F_{\mu \nu}$ and its derivatives times an appropriate power of $g$. We should then ask whether such terms contribute to the SW action (1.11). We find that their contribution has the form

$$
\int d^{3} x F^{\mu v}\left(\partial_{\mu} T_{v \alpha \beta}-\mu g^{2} \varepsilon_{\mu v \rho} T_{\alpha \beta}^{\rho}\right) .
$$

Let us now examine the first terms in the expansion of $T_{\mu \alpha \beta}$ in powers of $1 / g$. The leading terms are

$$
\frac{1}{g^{4}} \varepsilon_{\alpha \beta \rho} F_{\mu}^{\rho}, \quad \frac{1}{g^{4}} \varepsilon_{\mu[\alpha}{ }^{\rho} F_{\beta] \rho} .
$$

The first term can be removed by a gauge transformation and a rigid translation while for the second term (1.12) vanishes so both can be disregarded. The next terms have the form

$$
\frac{1}{g^{6}} \partial_{\mu} F_{\alpha \beta}, \quad \frac{1}{g^{6}} \partial_{[\alpha} F_{\beta] \mu},
$$

and again the first term can be removed by a gauge transformation while the second is proportional to the first after using the Bianchi identity. Higher order terms, however, can contribute. For instance, to order $1 / g^{8}$ we find that $\varepsilon_{\mu \alpha \beta} F^{2}$ gives a non trivial contribution since (1.12) does not vanish. Its contribution to the $\mathrm{SW}$ action (1.11) is

$$
-\frac{1}{g^{4}} \theta^{\alpha \beta} \varepsilon_{\alpha \beta \mu} F^{2} \partial_{v} F^{\mu v}-\frac{2 \mu}{g^{2}} \theta^{\alpha \beta} F_{\alpha \beta} F^{\mu v} F_{\mu \nu} .
$$

Notice that we get a contribution of order $1 / g^{2}$ and the coefficient of such a contribution could be chosen to cancel the corresponding term in (1.11).

The ambiguity found here is not of the same sort as that found by successive applications of the SW map [11]. Here it arises because the model has a dimensionfull coupling constant. If we require the SW map to be universal in the sense that it applies to any gauge theory then such terms are not present. We will take this point of view from now on.

In [13] the SW map for the NC Stückelberg-Proca theory has been obtained by requiring that in the unitary gauge it gives the Proca theory. Using the same criterion, the SW map for the NC Stückelberg-SD model is found to be

$$
\begin{aligned}
& \hat{f}_{\mu}=f_{\mu}-\frac{1}{2} \theta^{\alpha \beta} b_{\alpha}\left(2 \partial_{\beta} f_{\mu}-\partial_{\mu} b_{\beta}\right), \\
& \hat{b}_{\mu}=b_{\mu}+\frac{1}{2} \theta^{\alpha \beta} \partial_{\alpha} b_{\mu} b_{\beta}
\end{aligned}
$$


while the gauge parameter transforms as

$$
\hat{\alpha}=\alpha-\frac{1}{2} \theta^{\alpha \beta} b_{\alpha} \partial_{\beta} \alpha
$$

Applying the map to (1.8) we obtain the SW mapped action

$$
\begin{aligned}
L_{S W S D} & =\int d^{3} x \frac{g^{2}}{2}\left[\left(f_{\mu}-b_{\mu}\right)\left(f^{\mu}-b^{\mu}\right)+\theta^{\alpha \beta}\left(f_{\mu}-b_{\mu}\right)\left(2 b_{\alpha} \partial_{\beta} f_{\mu}-b_{\alpha} \partial_{\mu} b_{\beta}+\partial_{\alpha} b_{\mu} b_{\beta}\right)\right] \\
& -\frac{1}{4 k} \int d^{3} x \varepsilon_{\mu v \lambda} f^{\mu v} f^{\lambda}-\theta^{\alpha \beta} \varepsilon_{\mu v \lambda}\left[f^{\mu v} b_{\alpha}\left(2 \partial_{\beta} f^{\lambda}-\partial^{\mu} b_{\beta}\right)+\frac{4}{3} f^{\mu} \partial_{\alpha} f^{v} \partial_{\beta} f^{\lambda}\right] .(1
\end{aligned}
$$

\section{Equivalence of the MCS theory and the SD model}

In order to make the procedure of deriving the dual theory in NC space-time more transparent and also to set up our notation, we present a brief derivation of the well known equivalence between the MCS theory and the SD model in commutative space-time. The MCS theory described by the Lagrangian

$$
\mathscr{L}_{M C S}=-\frac{1}{4 g^{2}} F_{\mu v} F^{\mu v}+\frac{\mu}{2} \varepsilon_{\mu v \lambda} A^{\mu} \partial^{v} A^{\lambda},
$$

is invariant under the $U(1)$ gauge transformation $A_{\mu} \rightarrow A_{\mu}+\partial_{\mu} \alpha$ while the SD model, whose Lagrangian is

$$
\mathscr{L}_{S D}=\frac{g^{2}}{2} f_{\mu} f^{\mu}-\frac{1}{2 k} \varepsilon_{\mu \nu \lambda} f^{\mu} \partial^{v} f^{\lambda},
$$

has no such an invariance since the $f_{\mu} f^{\mu}$ term breaks the symmetry. Their equivalence has been analyzed using a phase space path integral approach [14] and it was shown that the SD model is equivalent to a gauge fixed version of MCS theory. Also, this equivalence has been been studied within the generalized canonical framework of Batalin and Fradkin in [15]. It was shown that the gauge invariant formulation obtained by the Hamiltonian embedding of SD model is equivalent to the $U(1)$ invariant MCS theory, clarifying the equivalence between both theories in spite of fact that they have different gauge structures. The procedure employed here also sheds light into this issue as we shall see.

The MCS theory is also invariant under a global shift of the vector field $A_{\mu} \rightarrow A_{\mu}+\xi_{\mu}$ apart from the $U(1)$ gauge invariance. We first elevate this global shift symmetry to a local one by gauging it by an appropriate antisymmetric gauge field $G_{\mu \nu}$ which transforms as $G_{\mu \nu} \rightarrow G_{\mu \nu}+$ $\partial_{\mu} \xi_{v}-\partial_{\nu} \xi_{\mu}$. To have the same physical content as our starting MCS theory we then constrain this gauge field to be non-propagating. This is done by introducing a Lagrange multiplier $\Phi$ which imposes the dual field strength of this gauge field to be flat. The result is

$$
\begin{aligned}
\mathscr{L} & =-\frac{1}{4 g^{2}}\left(F_{\mu \nu}-G_{\mu v}\right)\left(F^{\mu v}-G^{\mu v}\right)+\frac{\mu}{4} \varepsilon_{\mu \nu \lambda} P^{\mu}\left(F^{v \lambda}-G^{v \lambda}\right)-\frac{\mu}{8} \varepsilon_{\mu \nu \lambda} P^{\mu} \partial^{v} P^{\lambda} \\
& +\frac{1}{4} \varepsilon_{\mu \nu \lambda} G^{\mu v} \partial^{\lambda} \Phi+\frac{1}{4} \varepsilon_{\mu \nu \lambda} J^{\mu}\left(F^{v \lambda}-G^{v \lambda}\right),
\end{aligned}
$$

where we have introduced an auxiliary field $P_{\mu}$ to linearize the CS term. This field has a $U(1)$ gauge invariance $P_{\mu} \rightarrow P_{\mu}+\partial_{\mu} \chi$ when the multiplier field transforms as $\Phi \rightarrow \Phi+\mu \chi$ and $A_{\mu} \rightarrow A_{\mu}$. The last term in the Lagrangian is a source $J^{\mu}$ coupling to the local shift invariant combination of $A_{\mu}$ 
and $G_{\mu \nu}$. The MCS theory is recovered from the above Lagrangian by eliminating the $\Phi$ field using its equation of motion.

To show the equivalence to the SD model we start from the partition function

$$
Z=\int D \Phi D P_{\mu} D A_{\mu} D G_{\mu \nu} e^{-i \int d^{3} x \mathscr{L}} .
$$

Integrations over $G_{\mu \nu}$ and $A_{\mu}$ are Gaussian and can be done trivially leading to

$$
Z_{\text {dual }}=\int D \Phi D P_{\mu} e^{-i \int d^{3} x \mathscr{L}_{e f f}} .
$$

After the redefinitions $\mu P_{\mu}=f_{\mu}$ and $\Phi=\Lambda$, we get the effective Lagrangian

$$
\mathscr{L}_{\text {eff }}=\frac{g^{2}}{8}\left(f_{\mu}-\partial_{\mu} \Lambda\right)\left(f^{\mu}-\partial^{\mu} \Lambda\right)-\frac{1}{8 \mu} \varepsilon_{\mu \nu \lambda} f^{\mu} \partial^{v} f^{\lambda}+\frac{g^{2}}{8} J_{\mu} J^{\mu}+\frac{g^{2}}{4}\left(f^{\mu}-\partial^{\mu} \Lambda\right) J_{\mu} .
$$

This theory is invariant under the $U(1)$ gauge transformation $f_{\mu} \rightarrow f_{\mu}+\partial_{\mu} \alpha$ when the Stuickelberg field transforms as $\Lambda \rightarrow \Lambda+\alpha$. We also note that the MCS coupling constant $g^{2}$ and the ChernSimons parameter $\mu$ have both appeared as inverse couplings when compared with (2.2). We can now fix the gauge invariance in (2.6), for instance by choosing the unitary gauge $\Lambda=0$, to recover the self-dual model given in (2.2). We thus conclude that the $U(1)$ invariant MCS theory is dual to the $U(1)$ invariant Stückelberg formulation of self-dual model.

From the partition functions (2.4) and (2.5) we derive the mapping between the n-point correlators for these theories. For the 2-point function, we get

$$
\left\langle\varepsilon_{\mu \nu \lambda} F^{v \lambda}(x) \varepsilon_{\alpha \beta \rho} F^{\beta \rho}(y)\right\rangle \equiv g^{4}\left\langle\left(f_{\mu}-\partial_{\mu} \Lambda\right)(x)\left(f_{\alpha}-\partial_{\alpha} \Lambda\right)(y)\right\rangle+g^{2} g_{\mu \alpha} \delta(x-y),
$$

leading the identification (up to non-propagating contact terms) between the gauge invariant combinations

$$
\varepsilon_{\mu v \lambda} F^{v \lambda} \leftrightarrow g^{2}\left(f_{\mu}-\partial_{\mu} \Lambda\right)
$$

This equivalence between SD model and MCS theory has been extended to include interaction with matter [7]. It has been shown that the SD model minimally coupled to charged dynamical fermionic and bosonic matter fields is equivalent to a MCS theory non-minimally coupled to matter. In the weak coupling limit, it was shown in [16] that the non-Abelian MCS theory is equivalent to non-Abelian SD model and recently it was shown that, perturbatively, this equivalence exists in all regimes of the coupling constant [17].

After re-expressing the NCMCS theory (1.7) in terms of $A_{\mu}$ and $\theta^{\alpha \beta}$ using the SW map (1.1) we apply the above procedure to construct the corresponding dual theory. Then by comparing this dual theory with SW mapped NC Stuickelberg-SD model, we study the status of their equivalence. We take up this in the next section.

\section{Seiberg-Witten mapped Maxwell-Chern-Simons theory and duality}

By applying the SW map (1.1) to the NCMCS Lagrangian (1.7) we get to order $\theta$

$$
\begin{aligned}
\mathscr{L}_{S W} & =-\frac{1}{4 g^{2}}\left[F_{\mu v} F^{\mu v}+2 \theta^{\alpha \beta} F_{\alpha \mu} F_{\beta v} F^{\mu v}-\frac{1}{2} \theta^{\alpha \beta} F_{\alpha \beta} F_{\mu v} F^{\mu v}\right] \\
& +\frac{\mu}{4} \varepsilon_{\mu \nu \lambda} P^{\mu} F^{v \lambda}-\frac{\mu}{8} \varepsilon_{\mu \nu \lambda} P^{\mu} \partial^{v} P^{\lambda},
\end{aligned}
$$


where an auxiliary field $P_{\mu}$ was introduced to linearize the CS term. We have also used the fact that the NCCS term gets mapped to the usual commutative CS term by the SW map [12]. After rewriting the above Lagrangian using auxiliary fields $B_{\mu \nu}$ and $C_{\mu \nu}$ as

$$
\begin{aligned}
\mathscr{L}_{S W} & =-\frac{1}{4 g^{2}} C_{\mu v} B^{\mu v}-\frac{\mu}{8} \varepsilon_{\mu v \lambda} P^{\mu} \partial^{v} P^{\lambda}+\frac{\mu}{4} \varepsilon_{\mu v \lambda} P^{\mu} F^{v \lambda} \\
& -\frac{1}{4 g^{2}}\left[F_{\mu v} F^{\mu v}+2 \theta^{\alpha \beta} C_{\alpha \mu} C_{\beta v} F^{\mu v}-\frac{1}{2} \theta^{\alpha \beta} C_{\alpha \beta} C_{\mu v} F^{\mu v}-B_{\mu v} F^{\mu v}\right],
\end{aligned}
$$

we can now gauge the shift invariance of $A_{\mu}$ field as in the commutative case. Due to the introduction of $B_{\mu \nu}$ and $C_{\mu \nu}$ we see that $G_{\mu \nu}$ will appear quadratically and this will simplify the calculation considerably. So, we introduce a gauge field $G_{\mu \nu}$ to promote the global shift invariance of $A_{\mu}$ to a local one. We then get

$$
\begin{aligned}
\mathscr{L}_{S W} & =-\frac{1}{4 g^{2}} C_{\mu v} B^{\mu v}-\frac{\mu}{2 \cdot 4} \varepsilon_{\mu \nu \lambda} P^{\mu} \partial^{v} P^{\lambda}+\frac{\mu}{4} \varepsilon_{\mu \nu \lambda} P^{\mu}\left(F^{v \lambda}-G^{\nu \lambda}\right)-\frac{1}{4} \varepsilon_{\mu v \lambda} G^{\mu v} \partial^{\lambda} \Phi \\
& -\frac{1}{4 g^{2}}\left[\left(F_{\mu v}-G_{\mu v}\right)+\left(2 \theta^{\alpha \beta} C_{\alpha \mu} C_{\beta v}-\frac{1}{2} \theta^{\alpha \beta} C_{\alpha \beta} C_{\mu v}\right)-B_{\mu \nu}\right]\left(F^{\mu v}-G^{\mu v}\right) .
\end{aligned}
$$

Starting with the partition function

$$
Z=\int D P_{\mu} D \Phi D C_{\mu v} D B_{\mu \nu} D A_{\mu} D G_{\mu \nu} e^{-i \int d^{x} \mathscr{L}_{S W}},
$$

we can integrate over $G_{\mu \nu}, A_{\mu}$ and $B_{\mu \nu}$ to get the partition function corresponding to the effective Lagrangian

$$
\begin{aligned}
\mathscr{L}_{e f f} & =-\frac{\mu}{8} \varepsilon_{\mu v \lambda} P^{\mu} \partial^{v} P^{\lambda}-\frac{1}{4 g^{2}} C_{\mu \nu} C^{\mu v}+\frac{1}{4} \varepsilon_{\mu \nu \lambda} C^{\mu v}\left(\mu P^{\lambda}-\partial^{\lambda} \Phi\right) \\
& -\frac{1}{4 g^{2}} C^{\mu \nu}\left[2 \theta^{\alpha \beta} C_{\alpha \mu} C_{\beta v}-\frac{1}{2} \theta^{\alpha \beta} C_{\alpha \beta} C_{\mu v}\right] .
\end{aligned}
$$

We have neglected higher order terms in $\theta$ in performing the Gaussian integrals. It is easy to see that in the commutative limit we get (2.2) when $C_{\mu \nu}$ is eliminated by using its field equation and setting $\Phi=0$.

In the NC case $C_{\mu \nu}$ can be eliminated perturbatively in $\theta$. We then get

$$
\begin{aligned}
\mathscr{L}_{\text {dual }} & =\frac{g^{2}}{8}\left(f_{\mu}-\partial_{\mu} \Lambda\right)\left(f^{\mu}-\partial^{\mu} \Lambda\right)+\frac{g^{4}}{32} \theta^{\alpha \beta} \varepsilon_{\alpha \beta \lambda}\left(f^{\lambda}-\partial^{\lambda} \Lambda\right)\left(f^{\mu}-\partial^{\mu} \Lambda\right)\left(f_{\mu}-\partial_{\mu} \Lambda\right) \\
& -\frac{1}{8 \mu} \varepsilon_{\mu \nu \lambda} f^{\mu} \partial^{v} f^{\lambda},
\end{aligned}
$$

where we have identified $\mu P_{\mu}=f_{\mu}$ and $\Phi=\Lambda$. As in the commutative case the strong coupling limit of the original theory gets mapped into the weak coupling limit of the dual. It is easy to see that in the limit of vanishing $\theta$ the above Lagrangian (in the unitary gauge where $\Lambda=0$ ) correctly reproduces the SD Lagrangian (2.2).

It is interesting to note that the explicit form of the order $\theta$ term in the $C_{\mu v}$ field equation is not need at all to find the above Lagrangian. This happens because there are nice cancellations and it is 
easy to be convinced that to obtain the dual Lagrangian to $n$-th order in $\theta$ we need the perturbative solution for $C_{\mu \nu}$ only to order $(n-1)$.

We can couple a source term $\varepsilon_{\mu v \lambda} F^{\mu v} J^{\lambda}$ to the Lagrangian (3.1) and this leads to the map between the 2-point functions

$$
\begin{gathered}
\left\langle\varepsilon_{\mu v \lambda} F^{v \lambda}(x) \quad \varepsilon_{\alpha \beta \rho} F^{\beta \rho}(y)\right\rangle \equiv g^{4}\left\langle\tilde{f}_{\mu}(x) \tilde{f}(y)\right\rangle+g^{2} g_{\mu \alpha} \delta(x-y) \\
+\frac{g^{8}}{64}\left\langle\bar{\theta}_{\mu} \tilde{f}^{v} \tilde{f}_{v}+2 \bar{\theta}_{v} \tilde{f}^{v} \tilde{f}_{\mu} \bar{\theta}_{\alpha} \tilde{f}^{\beta} \tilde{f}_{\beta}+2 \bar{\theta}_{\beta} \tilde{f}^{\beta} \tilde{f}_{\alpha}\right\rangle+g^{4}\left(\bar{\theta}_{\mu} \tilde{f}_{\alpha}+\bar{\theta}_{\alpha} \tilde{f}_{\mu}+\bar{\theta}_{\beta} \tilde{f}_{\beta} g_{\mu \alpha}\right),
\end{gathered}
$$

where $\bar{\theta}_{\mu}=\varepsilon_{\mu \nu \lambda} \theta^{v \lambda}$ and $\tilde{f}_{\mu}=f_{\mu}-\partial_{\mu} \Lambda$. In the limit $\theta \rightarrow 0$ we recover the map obtained in (2.7).

Here we note that all the $\theta$ dependence of the SW mapped NCMCS theory comes from the Maxwell term alone as the NCCS term gets mapped to usual commutative CS term. Since it is possible to express the SW mapped Maxwell action to all orders in $\theta$ in terms of the commutative field strength $F_{\mu \nu}$ and $\theta$ alone [18](an exact closed form for the SW mapped Maxwell action is given in [19]), it is easy to convince from (3.1) and (3.3) that the procedure adopted here can be used to construct the dual theory to all orders in $\theta$ using a perturbative solution for the $C_{\mu v}$ field equation.

One important point to note is that the theory described by the Lagrangian (3.6), which is equivalent to the SW mapped NCMCS theory, is not the same as the SW mapped action for NCSD model (1.18). This clearly shows that the SW mapped theories are not equivalent.

\section{Conclusion}

In this paper we have constructed and studied the dual description of the NCMCS theory and investigated the status of the equivalence between this theory and SD model. We have derived the dual theory starting from the SW mapped NCMCS Lagrangian which is given in terms of commutative fields and the NC parameter. The equivalence was obtained at the level of partition functions and it allowed us to get the mapping between the n-point correlators of both theories. We have shown that the dual theory does not coincide with the SW mapped NC Stückelberg-SD theory. However, in the commutative limit, we recover the well known equivalence between them. We have also shown that the the two-point correlators map reduces to the one obtained in the commutative case in this limit. We have argued that this result can be extended to all orders in $\theta$ due to the structure of the SW mapped NCMCS Lagrangian. We have also verified that even after accounting for the ambiguous terms in the SW map, the dual theory and SW mapped NC Stückelberg-SD model are not equivalent.

Hence, we have shown that the equivalence between the MCS theory and the SD model in commutative space-time does not survive in the NC case. In this respect we are in agreement with the results obtained earlier in [3] and [6] where it was argued that these NC theories are not equivalent. But unlike the NCCS term used in [3], we have used the standard NC $U(1)$ invariant CS term with a cubic interaction as in [6] and [4]. The non-equivalence between the NCSD model and NCMCS theory shown here will come as an obstacle in generalizing the bosonization of the commutative Thirring model to NC space-time as was pointed out in $[3,6]$. 


\section{ACKNOWLEDGMENTS:}

The work of VOR was partially supported by CNPq, FAPESP and PRONEX under contract CNPq 66.2002/1998-99. This paper was done in collaboration with E. Harikumar

\section{References}

[1] E. Harikumar and V. O. Rivelles, "Noncommutative Maxwell-Chern-Simons theory in three dimensions and its dual,” Phys. Lett. B 625, 156 (2005) [arXiv:hep-th/0506078].

[2] S. Deser, R. Jackiw and S. Templeton, "Three-dimensional Massive Gauge Theories”, Phys. Rev. Lett. 48 (1982) 975; Ibid, “Topologically Massive Guage Theories”, Ann. Phys. 140 (1982) 372; S. Deser and R. Jackiw, "'Selfduality of Topologically Massive Gauge Theories”, Phys. Lett. B139 (1984) 371.

[3] S. Ghosh, "Gauge Invariance and Duality in the Noncommutative Plane”, Phys. Lett. B558 (2003) 245, hep-th/0210107; ibid, "Bosonization in the Noncommutative Plane", Phys. Lett. B563 (2003) 112 , hep-th/0303022.

[4] M. B. Cantcheff, P. Minces, "Duality in Noncommutative Topologically Massive Gauge Field Theory Revisited”, Eur. Phys. J. C34 (2004) 393, hep-th/0306206.

[5] N. Seiberg, E. Witten, “String Theory and Noncommutative Geometry”, JHEP 09, 032 (1999), hep-th/9908142.

[6] T. Mariz, R. Menezes, J. R. S. Nascimento, R. F. Ribeiro and C. Wotzasek, "Issues of duality on non-commutative manifolds: the non-equivalence between self-dual and topologically massive models", Phys. Rev. D70 (2004) 085018, hep-th/0306265.

[7] M. A. Anacleto, A. Ilha, J. R. S. Nascimento, R. F. Ribeiro and C. Wotzasek, "Dual equivalence between Self-Dual and Maxwell-Chern-Simons models coupled to dynamical U(1) charged matter", Phys. Lett. B504 (2001) 268, hep-th/0104152; M. Gomes, L. C. Malacarne and A. J. Silva, "On the equivalence of the self-dual and Maxwell-Chern-Simons models coupled to Fermions", Phys. Lett. B439 (1998) 137, hep-th/9711184.

[8] M. S. Guimarães, J. L. Noronha, D. C. Rodrigues and C. Wotzasek, "On duality of the noncommutative extension of the Maxwell-Chern-Simons model”, Phys. Lett. B605 (2005) 419, hep-th/0410156.

[9] T. H. Buscher, “Symmetry Of The String Background Field Equations”, Phys. Lett. B194 (1987) 59; ibid "Path Integral Derivation Of Quantum Duality In Nonlinear Sigma Models", Phys. Lett. B201, (1988) 466; U. Lindström and M. Rocek, Scalar Tensor Duality and $N=1, N=2$ Nonlinear Sigma Models", Nucl. Phys. B222 (1983) 285; N. Hitchin, A. Karlhede, U. Lindström and M. Rocek, "Hyper Kahler Metrics and Supersymmetry”, Commun. Math. Phys. 108 (1987) 535.

[10] E. Harikumar and M. Sivakumar, "Duality and Massive Gauge Invarinat Theories”, Phys. Rev. D 57 (1998) 3794, hep-th/9604181.

[11] T. Asakawa and I. Kishimoto, "Comments on Gauge Equivalence in Noncommutative Geometry", JHEP 9911 (1999) 024, hep-th/9909139.

[12] N. Grandi and G.A. Silva, "Chern-Simons Action in Noncommutative Space”, Phys. Lett. B507 (2001) 345, hep-th/0010113. 
[13] R. Amorim, N. R. F. Braga and C. N. Ferreira, "Nonequivalent Seiberg-Witten maps for noncommutative massive U(N) gauge theory”, Phys. Lett. B591 (2004) 181, hep-th/0312089.

[14] R. Banerjee, H. J. Rothe and K. D. Rothe, "On the Equivalence of the Maxwell-Chern-Simons Theory and a Self-Dual Model”, Phys. Rev. D52 (1995) 3750, hep-th/9504067.

[15] R. Banerjee, H. J. Rothe and K. D. Rothe, "Hamiltonian Embedding of Self-Dual Model and Equivalence with Maxwell-Chern-Simons Theory”, Phys. Rev. D55 (1997) 6339, hep-th/9611077.

[16] N. Bralić, E. Fradkin, V. Manias and F. A. Schaposnik, "Bosonization of Three Dimensional Non-Abelian Fermion Field Theories”, Nucl. Phys. B446 (1995) 144, hep-th/9502066.

[17] M. B. Cantcheff, "Parent Action Approach for the Duality between Non-Abelian Self-Dual and Yang-Mills-Chern-Simons Models”, Phys. Lett. B528 (2002) 283, hep-th/0110211.

[18] O. J. Ganor, G. Rajesh and S. Sethi, "Duality and Non-Commutative Gauge Theory", Phys. Rev. D62 (2000) 125008, hep-th/0005046; O. F. Dayi, "Noncommutative Maxwell-Chern-Simons theory, duality and a new noncommutative Chern-Simons theory in $d=3$ ”, Phys. Lett. B560 (2003) 239, hep-th/0302074.

[19] R. Banerjee and H. S. Yan, "Exact Seiberg-Witten Map, Induced Gravity and Topological Invariants in Noncommutative Field Theories", Nucl. Phys. B708 (2005) 434, hep-th/0404064. 